\title{
Differentiation of cochlear neural progenitors with SV40 in vitro
}

\author{
Ling Feng ${ }^{1^{*}}$, Yuki Hamajima ${ }^{1^{*}}$, Masahiro Komori ${ }^{1}$, John Anderson ${ }^{1,2}$, Dong Wang ${ }^{5}$, Terry C. \\ Burns $^{2,3,4}$, Catherine M. Verfaillie ${ }^{4}$, Walter C. Low ${ }^{2,3,4}$ and Jizhen Lin ${ }^{1,4}$
}

\author{
Departments of ${ }^{1}$ Otolaryngology and ${ }^{2}$ Neurosurgery, ${ }^{3}$ Graduate Program in Neuroscience, \\ ${ }^{4}$ Stem Cell Institute, and ${ }^{5}$ Biomedical Engineering, University of Minnesota, Minneapolis, \\ Minnesota
}

*These authors contributed equally to this work.

\begin{abstract}
Sphere-forming cochlear stem cells/progenitors have recently been isolated from the postnatal mouse organ of Corti. However, self-renewal of the cochlear progenitors remains to be elucidated. Here we demonstrate the renewable cochlear neural progenitors from the postnatal Immortomouse organ of Corti containing a temperature-sensitive SV40 that have the potential to differentiate into hair cell-, neuron-, and supporting cell-like phenotypes under the guidance of sonic hedgehog, epidermal growth factor, retinoic acid, and brain-derived neurotrophic factor, herein termed SERB. This work provides a rationale for using cochlear neural progenitors in possible cell replacement of lost hair cells and neurons in degenerative hearing disorders.
\end{abstract}

Keywords: Cochlear neural progenitor; SV40; Growth factor; Differentiation; Mouse

\section{Introduction}

Chochlear sensory epithelial cells, capable of differentiating into hair cells, have been successfully isolated from the rat postnatal day 0 ( $\mathrm{P} 0)$ organ of Corti (1) and transgenic mouse P14 organ of Corti (2). Recently, sphere-forming cells with characteristic features of stem cells or progenitors, have been isolated from the mouse P1 P21 organ of Corti $(3,4)$ and the adult mouse utricle (5). However, self-renewal of the above stem cells/progenitors remains to be elucidated.

Received 11/17/08; accepted 02/04/09

Correspondence: Jizhen Lin, MD, University of Minnesota, 216 Lions Research Building. 2001 Sixth Street S.E.

Minneapolis, MN 55455, USA. Tel. 612-626-9872, Fax. 612-626-9871. email: linxx004@tc.umn.edu
The organ of Corti is known for its quiescence in cellular proliferation after the late-term embryonic stage, which forms a non-proliferating zone (6). This may involve a variety of inhibitory genes (or cell cycle inhibitors) such as retinoblastoma protein $(\mathrm{Rb})$ (7) that creates an adverse situation for progenitor proliferation. Knockout of $\mathrm{Rb} 1$ protein in mice demonstrated the extra hair cell numbers in the organ of Corti (8), adding support to the notion that proliferation of progenitors is inhibited in the mammalian cochlear system. Therefore, exogenous stimuli of growth factors and cytokines may be needed to remove inhibition of $\mathrm{Rb} 1$ for $\mathrm{CNPs}$ to continue to self-renew in the mammalian organ of Corti after birth.

If this assumption is correct, an oncogene such as SV40 would overcome the inhibition by $\mathrm{Rb}$ protein. The viral protein SV40 large $\mathrm{T}$ antigen contains an amino acid motif ( $\mathrm{LxCxE}$ ), which is able to bind the conserved pocket region of an $\mathrm{Rb}$ protein (7) that disables the function of Rb. We hypothesized in this study that SV40 is able to extend life-span of cochlear neural progenitors (CNPs ) in vitro in an unlimited manner. To test this hypothesis, Immortomouse at $\mathrm{P} 1$ was used as donors of CNPs in this study. To make CNPs from Immortomouse inducible for differentiation, a temperature $\left(37^{\circ} \mathrm{C}\right)$ close to non-permission $\left(39^{\circ} \mathrm{C}\right)$, was employed for culture of cochlear neural progenitors. To induce differentiation of cochlear neural progenitors, a group of growth factors (SERB), defined in our recent study (4), was used.

Sonic hedgehog (SHH) is involved in the development of the inner ear (9). Retinoic acid (RA) stimulates the regeneration of mammalian auditory hair cells (10). Epidermal growth factor (EGF) is a growth factor for inner ear utricle stem cells (5). 
Brain-derived neurotrophic factor (BDNF) is a surviving factor for cochlear sensory epithelial cells in rats (11). Neuroepithelial cell differentiation usually occurs in the post-mitotic stage. To induce cellular differentiation, SERB was used to prime CNPs for 14 days and then withdrawn from culture media for 7 days in order to allow CNPs to differentiate. It was found that CNPs from the Immortomouse strain (positive for SV40) demonstrated an unlimited life-span of CNPs in vitro and an increase of hair cell markers in CNPs via the above two-step procedures. Importantly, resulting cells demonstrated hair bundle-like structures, which is an indication for profound differentiation.

\section{Materials and Methods Culture Media}

DMEM/F12 + 1\% N2 (Gibco) was used as basal media for CNPs in this study. N2 contains $25 \mu \mathrm{g} / \mathrm{mL}$ bovine insulin, $100 \mathrm{\mu g} / \mathrm{mL}$ transferring, $20 \mathrm{nM}$ progesterone, $60 \mu \mathrm{M}$ putrecine and $30 \mathrm{nM}$ sodium selenite. CNPs from C57BJ/6 Immortomouse were maintained and propagated in growth media that is consisted of basal media plus $20 \mathrm{ng} / \mathrm{mL}$ of EGF and $20 \mathrm{ng} / \mathrm{mL}$ of bFGF. For induction of differentiation, CNPs were incubated with basal media plus SERB for 14 days, followed by without any growth factors for 7 days. For isolation of single progenitor clones, growth media were used as previously described (4). Neural precursor cells (NPCs) from embryonic mouse brain were cultured as previously described (12).

\section{Isolation and maintenance of $C N P S$}

The animal use protocol was approved by the Institutional Animal Care and Use Committee (IACUC) at the University of Minnesota. C57BL/6 mice were used as donors in this study. This strain was chosen because it contains a temperaturesensitive SV40 (e.g., SV40 tsA58 TAg) that makes cells immortalized in vitro at $33^{\circ} \mathrm{C}$ in the presence of interferon (13). However, SV40 tsA58 TAg is inactivated when cultured at $39^{\circ} \mathrm{C}$ in the absence of interferon. Procedures for procurement of cells from the postnatal organ of Corti were performed as previously described (14), with some modifications. Briefly, two organ of Corti specimens from one P1 Immortomouse was dissected out from the both cochleas under an operating microscope and placed into $1 \mathrm{~mL}$ of growth media. Cells in the specimens were physically dissociated by pipetting, placed into two $35-\mathrm{mm}$ cell culture dishes, and cultured in an incubator with $5 \% \mathrm{CO}_{2}$ at $37^{\circ} \mathrm{C}$. Media were changed every other day. After 3-5 day culture, cells in floating or loosely adherent were physically removed and transferred to new culture dishes. Within 5-7 days, single cells gradually grew into multiple cellular spheres. Cells in cellular balls were dissociated by pipetting, and divided into new plates every 5-7 days at a cell density of 7-10 small balls per $35 \mathrm{~mm}$ dish. Isolation of CNPs from mice was repeated three times.

\section{Characterization of primitive CNPS}

After establishment of cochlear neural progenitors, 10-100 single cells were cultured on 8well chamber slides with $5 \mu \mathrm{M}$ of bromodeoxyuridine (BrdU) added to growth media at the beginning of experiment. Cellular location of single cells was marked by a marker pen and documented under a phase-contrast microscope for clonal growth on days 1, 3, and 5. Media changed every other day. Cells were fixed with $100 \%$ ethanol at room temperature for 6 minutes on days 1,3 , and 5, respectively. Cellular balls on the chamber slides were stained with anti-BrdU antibody (sheep IgG, ab1893) and secondary antibody (donkey anti-sheep, Santa Cruz) conjugated to TRITC for evaluating proliferating cells and then counter-stained with DAPI for cellular nuclear stain.

For examination of cellular marker expression in cellular balls, clonal CNPs were cultured on 8-well chamber slides for 7-10 days, fixed with $100 \%$ ethanol for 6 minutes at room temperature, preincubated with $3 \%$ normal donkey serum for 15 minutes, incubated with primary and secondary antibodies sequentially. Double immunohistochemistry was performed for cellular marker coexpression. Briefly, cell culture slides were incubated with a primary antibody, washed with $0.2 \%$ detergent (TWEEN) in PBS, incubated with a secondary antibody conjugated to fluorescein isothiocyanate (FITC, green) or tetramethylrhodamine isothiocyanate (TRITC, red), washed again, washed in $0.2 \%$ TWEEN in PBS for 5 minutes, and observed under a confocal or fluorescent microscope. Chamber slides incubated with non-specific IgG (Zymed) served as controls. Primary antibodies used in this study were nestin (BD Pharmingen, 1:100); glial fibrillary acidic protein (GFAP, DakaCytomation, 1:500); SRY-box containing gene 2 (Sox2, Sigma, 1:400), Math1 (Abcam, 1:100); espin (BD Bioscience, 1:400), and myosin VIIa (Sigma, 1:100). Secondary antibodies used were FITC or TRITC-conjugated mouse anti- 
rabbit, goat anti-mouse IgG (Zymed), or pholloidin (TRITC-conjugated, Sigma). Counter-stain with 4',6diamidino-2-phenylindole (DAPI) was performed on some slides.

For cell marker expression, reverse transcription polymerase chain reaction (RT-PCR) was performed for primitive NPCs and cochlear neural progenitors; cellular balls, and CNPs at $10^{\text {th }}$ passage. Specific primers for the Math1 (5'-agatctaca tcaacg ctctgtc3'/5'-actggcctcatcagagtcactg-3', 452 bp), Brn3.1 (5'ctctggcggcggtggata-3'/5'-acggcat gcgggtgactc-3', 324 bp), espin (isoforms 1, 2, 3, and 6, 5'cagcctgagtcaccgcagctc- 3 ' $/ 5$ ' - tgacctgtcgct gccagggegcg-3', 474 bp), myosin VIIa (5'aagcactgctcetgctcgtccacg-3'/5'-ctcctctacatcgctctgttc g-3', 628 bp), p19ink4d (5'-gcagtgtgactcaagagaagc-3'/5'gccacattcgctaagtgctcg-3', 1134bp); p27Kip1 (5'-ctggag cggatggacgccagac-3' /5'-cgtctgctccacagtgccagc-3'); Id1 (5'-ctacgacatgaacggetgctac-3' /5'-ctggggaaccgagagca ctt tt-3'); nestin (5'-gccg agctggagcgcgagttagag-3'/5'gcaagggggaag agaaggatgtcg-3', $696 \mathrm{bp}$ ), Sox1 (5'cacaactcggagatcagcaa-3'/5'-tgtaatccgggtgttccttc-3', $187 \mathrm{bp}$ ); Sox2, (5'-aggaaggagtttattcggatttg-3'/5'-acgat atcaacctgcatggac-3'; $181 \mathrm{bp}$ ) Musashi (Msi, 5'caagaaagctcagc caaagg-3' /5'- cggggaactggtaggtgtaa3'; 191 bp), Paired box gene 2 (Pax2, (5'ccaaagtggtggacaagattgcc-3' /5'-ggataggaaggacgct caaagac-3'); Pax 6 (5'-gtccatctttgcttgggaaa-3' $/ 5^{\prime}$ tagccaggttgcgaagaact-3', 111 bp); Otx2 (5'ccatgacctatactcaggcttcagg-3' /5'-gaagctccatatcctgggt ggaaag-3'); GFAP (5'-gaggagtggtatcggtctaagttttttg 3'/5'-cgggctctagggactcgtt-3', $165 \mathrm{bp}$ ); cytokeratin 18 (CK18, 5'-ggtgaagagcetggaaactg-3'/5'-ggtactctcctcaa tctgct-3'); collagen iv alpha 6 (COL4A6, 5'caggtttgtcaggcgtcact-3'/5'-tcgtggtgggtgatggggctttt-3'); neurogenin 1 (Ngn1, 5'-ccgacctccaaacctcctgt-3'/5'-ga tgtagttgtaggcgaagcgc-3'); NeuroD (5'-acgaggaac acgaggcagac-3'/5'-agtgggctgggacaaacctt- $\left.3^{\prime}\right)$; and Brn3a (5'-cccctcccaccttgttcgtt- 3 '/5'-acccagagtgagac ctaatc- $\left.3^{\prime}\right)$; and SV40 (5'-gcttggctacactgtttgttgc- $3^{\prime} / 5^{\prime}-$ tgtttcatgccctgagtcttcc- $3^{\prime}$ ) were verified using Amplify 1.2 software for selected primers regarding primer pair quality. Specificity of the primers was confirmed by BLAST search using the National Center of Biologic Information website (fttp://www.ncbi.nlm.nih.gov). RT-PCR was performed as described previously (14, 15). PCR products were analyzed on a $2 \%$ agarose gel and routinely purified for sequence verification. RNA samples omission served as controls.

For analysis of neuroepithelial cell marker profiles and growth potential on the early and late passges, cells from the $5^{\text {th }}$ and $45^{\text {th }}$ passage were cultured in flasks and grown in growth media with addition of $5 \mu \mathrm{M}$ of $\mathrm{BrdU}$ for 4 days and then harvested for evaluation of BrdU incorporation and neuroepithelial cell marker expression by double staining FACS. Semi-quantitative RT-PCR was performed as above for evaluation of mRNA expression (Id1, nestin, GFAP, Sox1, Sox2, Msi, Pax2, Pax6, espin, myosin VIIa, Math1, Brn3.1, p19ink4d). FACS was performed as previously described. Specific antibodies used for this study were Id1 (Santa Cruz, 1:100), Bmi-1 (Santa Cruz, 1:200), Sox2 (1:400), GFAP (1:500), and BrdU (abcam, 1:50), in a single- or double-staining manner. $\mathrm{Bmi}^{-1}$ was selected because it is a neural stem cell marker for self-renewal (16).

\section{Clonal CNPs and their proliferation features}

From the $5^{\text {th }}$ passage culture of primitive CNPs after enrichment, approximately 30 single cells were diluted in $2 \mathrm{~mL}$ of growth media, divided into 90 wells $(200 \mu \mathrm{L}$ per well) of a 96-well plate, and cultured in growth media until appearance of cell clones, as previously described (14). Growth of single clones was examined under a contrast microscope on a daily basis. Single clones were counted and documented. After that, 40,000 clonal progenitors were placed in each T-25 flask for 16 hours, with addition of $5 \mu \mathrm{M}$ of BrdU for evaluation of their proliferation for 4 days, and then harvested for expression of basic neuroepithelial cell marker expression by FACS (Id1, nestin, Sox2, GFAP, and $\mathrm{BrdU})$ in a single or double staining manner.

\section{Induction of hair cell marker in clonal progenitors}

Induction of hair cell markers were determined by RT-PCR and fluorescence-activated cell sorting (FACS). For RT-PCR, cells were cultured in flasks beginning at $4 \times 10^{5}$ cells/per flask, treated with and without EGF+bFGF, SHH, EGF, RA, BDNF, FGF19, and SERB for 4 days and harvested for RT-PCR and FACS. Briefly, total RNA was isolated from the above harvested cells using RNA Miniprep Kit (Stratagene). Residual genomic DNA in total RNA samples was digested with DNases according to the manufacturer's instruction. Specific primers for the myosin VIIa, Math1, and Brn3.1 genes were used (see above).

For analysis of neuroepithelial cell marker profiles and growth potential on the early and late passges, cells from the $5^{\text {th }}$ and $45^{\text {th }}$ passage were cultured in flasks and grown in growth media with addition of $5 \mu \mathrm{M}$ of $\mathrm{BrdU}$ for 4 days and then harvested for evaluation of BrdU incorporation and 
neuroepithelial cell marker expression by double staining FACS (BrdU/Id1, BrdU/Bmi-1, and nestin/Id1). Semi-quantitative RT-PCR was performed as above for evaluation of mRNA expression (Id1, nestin, GFAP, Sox1, Sox2, Msi, Pax2, Pax6, espin, myosin VIIa, Math1, Brn3.1, p19ink4d).

\section{Differentiation of clonal progenitors}

Differentiation of CNPs is usually associated with hair cell marker expression. To this end, differentiation of CNPs was determined by FACS, immunohistochemistry, and RT-PCR. For evaluation of hair cell marker expression, cells were cultured in flasks, treated with and without SERB for 14 days, then incubated with basal media (without SERB) for 7 days, and harvested for evaluation of myosin VIIa, Math1, B3-tubulin, and MAP2 by FACS, as described above.

For immunohistochemistry, clonal progenitors were cultured on 8-well chamber slides with SERB treatment for 14 days and without SERB for 7 days, fixed with $100 \%$ ethanol for 6 minutes at room temperature, pre-incubated with $3 \%$ normal donkey serum for 15 minutes, incubated with primary and secondary antibodies sequentially. Briefly, cell culture slides were incubated with a primary antibody, washed with $0.2 \%$ detergent (TWEEN) in PBS, incubated with a secondary antibody conjugated to green (FITC) or red (TRITC) fluorescein, washed again, incubated with DNA dye (DAPI, $0.7 \mathrm{\mu g} / \mathrm{mL}$ ) for 5 minutes, washed in $0.2 \%$ TWEEN in PBS for 5 minutes, and observed under a confocal or fluorescent microscope. Chamber slides incubated with non-specific IgG (Zymed) served as controls. In a similar manner, double-labeling immunohistochemistry was performed, as previously described (15).

Primary antibodies used in this study were Math1 (Abcam, 1:100); espin (BD Bioscience, 1:400); myosin VIIa (Sigma, 1:100); B3-tubulin (Sigma, 1:400); MAP2 (Sigma, 1:500); Id1 (Santa Cruz, 1:400); p27Kip1 (NeoMarker, mouse monoclonal, 1:200); S100A1 (DAKO, 1:200); and secondary antibodies used were FITC or TRITC-conjugated mouse anti-rabbit, goat anti-mouse IgG (Zymed), or pholloidin (TRITC-conjugated, Sigma). MAP2 and 83-tubulin double-positive cells were identified as immature neurons (63-tubulin is considered an immature neuronal marker though is also present at lower levels in mature neurons; MAP2 is a more mature neuronal marker); Math1 and myosin VIIa double-positive cells were identified as immature hair cells; whereas espin and rhodamine phalloidin (conjugated to TRITC) double-positive structures were identified as hair cell bundles.

\section{Statistics}

The differences between controls and experiments in cell counts, immunohistochemistry, and cell marker positive counts were calculated using unpaired student $t$ tests when two groups of experiments were involved and/or using ANOVA when multiple groups of experiments were involved. The confidence level at $95 \%$ was taken as being significant.

\section{Results \\ Isolation of CNPs from the P1 organ of Corti}

To isolate cochlear neural progenitors, C57BL/6 Immortomouse was used. The Immortomouse contains the tsSV40 oncogene that is sensitive to a temperature change. This temperature sensitive oncogene drives cell proliferation ultimately at $33^{\circ} \mathrm{C}$ (a permission temperature) when tsSV40 is fully active in function and not so at $39^{\circ} \mathrm{C}$ (a nonpermission temperature) when tsSV40 is fully inactive in function (Supplemental data 1). The organ of Corti was dissected out from three P1 mice using an operating microscope and three independent cultures were obtained from 3 mice. Cells from the organ of Corti were physically dissociated, and plated onto two $35-\mathrm{mm}$ culture dishes with $1 \mathrm{~mL}$ of growth media and cultured at $37^{\circ} \mathrm{C}$ that is in-between non-permissive and permissive temperature. In our earlier studies, this serum-free growth media favored growth of NPCs from the neonatal brain (12). The media contains no B27, leukemia inhibitory factor (LIF), or insulin-like growth factor (IGF-1), compared with previously used media for culturing otospheres $(1,3,17)$. It is believed that growth media are appropriate for growth of immature neuroepithelial cells such as CNPs or cochlear stem cells but not so for differentiated cells (non-progenitors or stem cells). Thus, growth media are thought as being selective for cochlear neural progenitors. Progenitor cultures reached approximately $40 \%$ confluence within one week in growth media. In these primary cultures, some cells $(5.5 \pm 3.6 \%, \mathrm{n}=3)$ were suspended in the culture media or loosely adherent to culture dishes, while the remaining were firmly adherent. Under a contrast microscope, the floating cells were small, round, and bright (Fig. 1a). Approximately $32.5 \pm 6.3 \%$ of cells $(n=3)$ remained non-adherent and developed into cellular spheres within 5 days (Fig. 
1b) in growth media at $10-100$ single cells $/ \mathrm{mm}^{2}$, while remaining cells adhered to the culture dish surface during the culture. Within a cellular ball, many of the cells were newly proliferated (BrdU positive, Fig. 1c)

The cellular balls were positive for neuroepithelial cell markers: nestin, GFAP, Sox2, Math1, espin, and myosin VIIa (Fig. 1d-1). In order to enrich cochlear neural progenitors, these floating or loosely adherent cells were removed and transferred to new culture dishes. After that, enriched CNPs were characterized by semiquantitative RT-PCR and found to express Id1, nestin, Sox1, Sox2, Msi, Pax6, GFAP, Pax2, Math1, Brn3.1, myosin VIIa, espin, but not p19ink4d which is expressed in differentiated hair cells. Id1 and nestin were highly expressed in CNPs compared with NPCs; Pax2 were expressed relatively abundant in CNPs but not so in NPCs; while Sox1, Sox2, Pax6, GFAP, and Brn3.1 were expressed relatively abundant in NPCs compared with CNPs by semiquantitative RT-PCR (Fig. 2A). Expression of several genes such as myosin VIIa and espin were relatively abundant in passaged CNPs compared with primitive CNPs whereas the expression of both Brn3.1 and Math1 was relatively weak in passaged CNPs compared with primitive CNPs (Fig. 2B). It has been noted that Brn3.1 and Math1 are abundantly expressed in NPCs.

Cells from adherent and/or loosely attached could be kept in an unlimited manner ( $>45$ passages at the time point of this report), yielding CNPs consistently (a mixture of both floating and adherent cells). A higher percentage of cells tended to be adherent when cultures were maintained at a high density but the potential to form cellular balls remained. Cultures were kept on a 5-7-day passage basis with growth media changed every other day. To compare the marker profile, CNPs at the $5^{\text {th }}$ and $45^{\text {th }}$ passages were compared with each other using FACS and semi-quantitative RT-PCR. It was found that the marker profile was similar between the $5^{\text {th }}$ and $45^{\text {th }}$ passages (Fig. 2C-D).

A question raised here is whether adherent cells are cochlear neural progenitors. To answer this question, adherent cells at the $5^{\text {th }}$ passage were subjected to continuous passages. It was found that adherent cells after passage yielded floating cells (Fig 3a). This suggests that adherent cells are cochlear neural progenitors. CNPs are believed to actively proliferate. Thus, formation of cellular spheres or balls within days is possible. To study this, CNPs from the primary and cultures subjected to multiple passages were grown into cellular balls in growth media (Fig. 3b). It was found that the capability to form cellular balls was observed in CNPs from both types of cultures (Fig. 3c-d) although the size for cellular balls from the primary cultures was bigger than that from the cultures subjected to multiple passages.

\section{Clonal progenitors and their proliferation features and neuroepithelial cell marker expression}

Clonal analysis is necessary for proving multipotency of cochlear neural progenitors. To this end, single progenitors were obtained from the $5^{\text {th }}$ passage (adherent cochlear neural progenitors) using a limiting dilution method as previously described (14). Wells containing a single cell were marked for subsequent analysis. A single cell alone
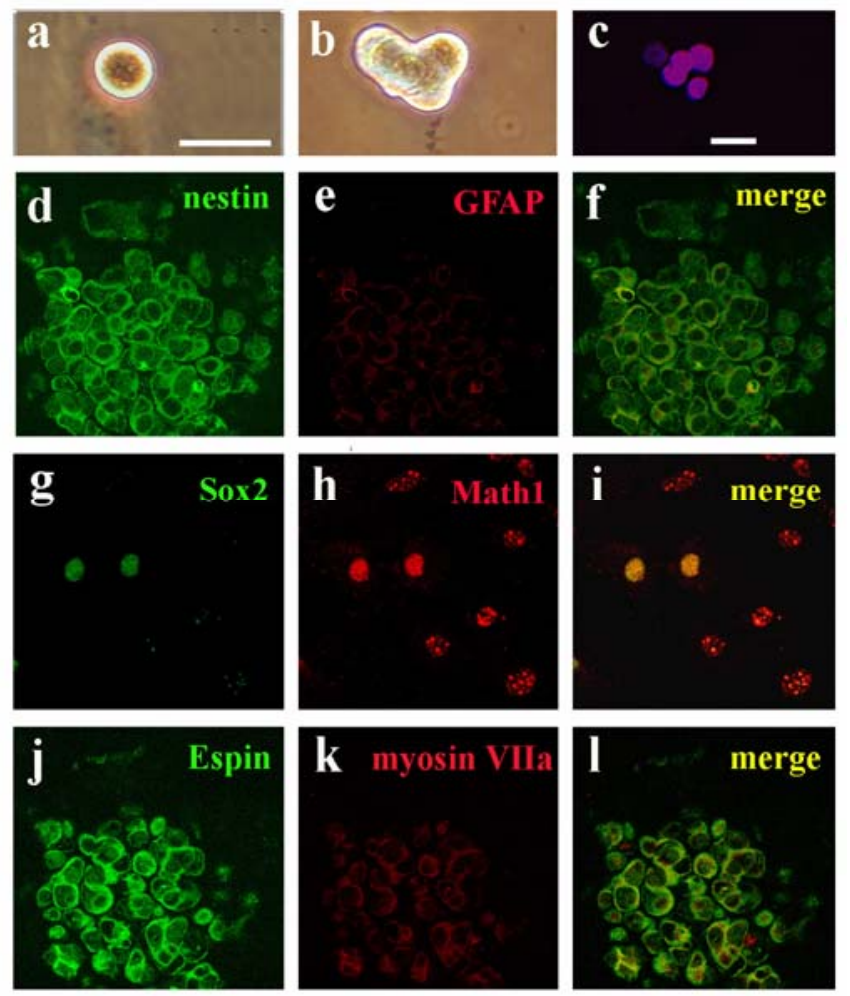

Figure 1. Identity of primitive cochlear neural progenitors (CNPs) from the mouse P1 organ of Corti. Phase-contrast micrograph showed small, round, and bright cells in the secondary culture (a) which was derived from a primary culture of the P1 Immortomouse organ of Corti. That cell expanded into a small cell ball within 5 days (b, a secondary culture) via a proliferative manner (c, BrdU positive in the majority of cells within the ball). Immunohistochemistry demonstrated cellular balls were positive for both nestin and GFAP (d-f). Sox2 and Math1 ( $\mathrm{g}-\mathrm{i})$, as well as espin and myosin VIIa (j-I). Note that relative weak expression of GFAP and myosin VIla and few cells positive for Sox2 in cellular balls (d-f and $\mathrm{j}-\mathrm{I}$ ) or adherent cells ( $\mathrm{g}-\mathrm{i})$. Bar=10 $\mu \mathrm{m}$ in (a) applying to (a-b) and in (c) applying to (c-I). 


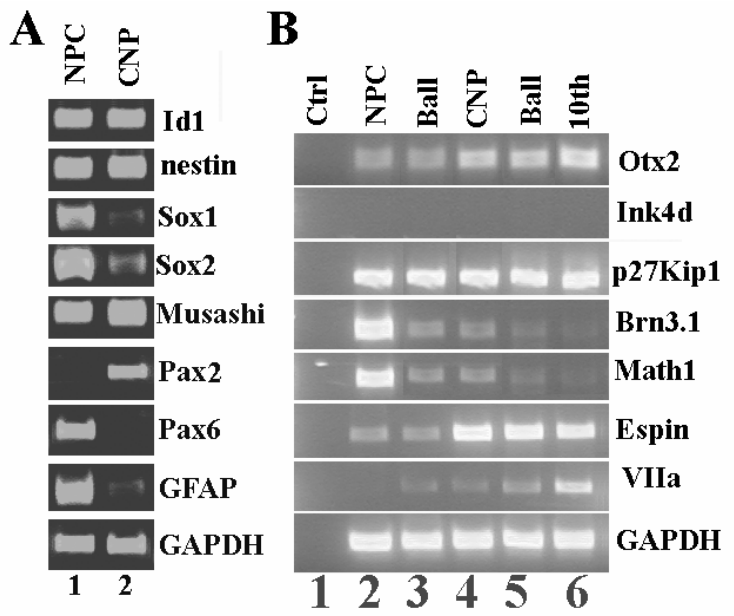

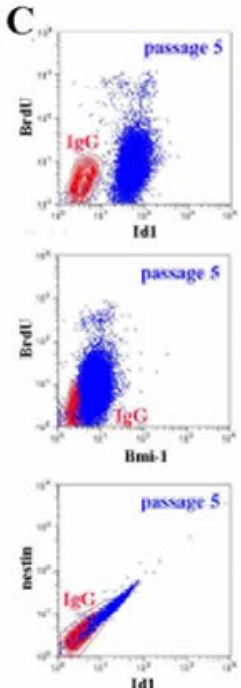
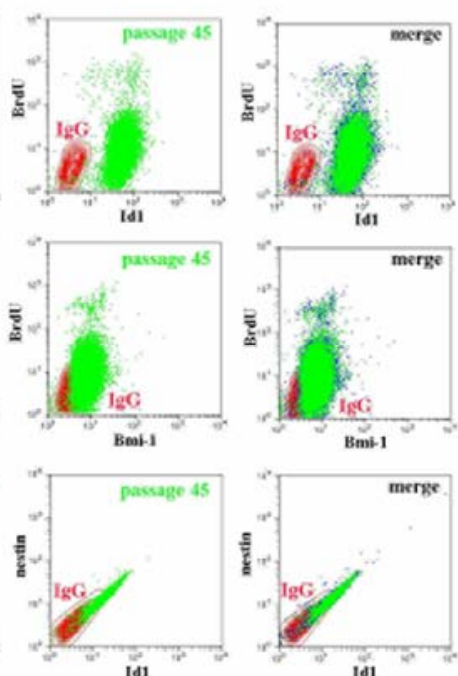

D

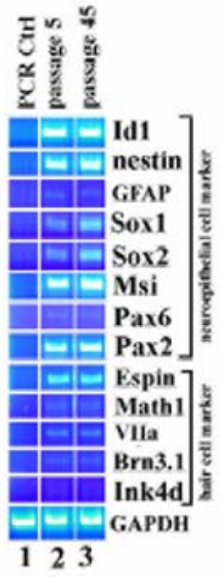

Figure 2. (A-B) Expression of neuroepithelial/hair cell markers between primitive NPCs and CNPs. In a transferred cell culture (A) from the primary culture of the P1 organ of Corti, CNPs expressed neuroepithelial cells markers by RT-PCR: Id1, nestin, Sox1, Sox2, Musashi, Pax2, Pax6, and GFAP as well as hair cell markers: Otx2, p19 ${ }^{\text {ink4d }}$, p27 ${ }^{\text {kip1 }}$, Brn3.1, Math1, espin, and myosin VIla. Expression of Sox1, Sox2, Pax6, and GFAP was weak in CNPs (lane 2) compared with NPCs (lane 1). While in a series of cultures (B) including a transferred CNP culture (lane 4), cellular balls (lane 5 ) after the transferred culture, and the subsequent $10^{\text {th }}$ passage (lane 6 ), hair cell markers such as Otx2, p2 $7^{\text {Kip1 }}$, Brn3.1, Math1, espin, and myosin VIla were expressed compared with neural procursor cells (NPC, lane 2) and their cellular balls (lane 3). It is noted that Brn3.1 and Math1 are weakened whereas Otx2 and myosin VIla are strengthened in cellular balls and subsequent passages. (C-D) Expression of neuroepithelial/hair cell markers in clonal CNPs. In clonal cell cultures from the $5^{\text {th }}$ to $45^{\text {th }}$ passages, cellular proliferation of CNP cultures was evaluated by FACS using BrdU incorporation and Id1/Bmi-1 expression (C) and cellular markers were evaluated by RT-PCR using neuroepithelial and hair cell markers (D). It was found that BrdU positive cells and Id1/Bmi-1 positive cells were similar between the $5^{\text {th }}$ to $45^{\text {th }}$ passages $(C)$ as well as neuroepithelial cell markers were equally expressed in the $5^{\text {th }}$ to $45^{\text {th }}$ passaged cultures (D).

was able to grow in growth media. Nineteen clones were established via this method from two 96-well plates, all of which had similar morphology. One of the clones (Clone \#5) was chosen for further analysis because they expressed abundant immature neuroepithelial cell markers: Id1 and nestin coexpression (Fig 4a-c), Sox2, and GFAP (Fig 4d). Cells were consistently adherent after clonal cultures. However, the clonal cultures were capable of continuing to proliferate as non-clonal progenitors, passage after passage, and proliferating cells (BrdU positive) were also Id1 positive and Id1 positive cells were also nestin positive (Fig. 4e). These cells remained to be adherent for most cultures but some (approximately $5 \%$ of cells) formed small cellular balls within 7-10 days in a similar way as primary cell cultures, similar to what was observed in nonclonal progenitor cultures (referred as to Fig. 3).
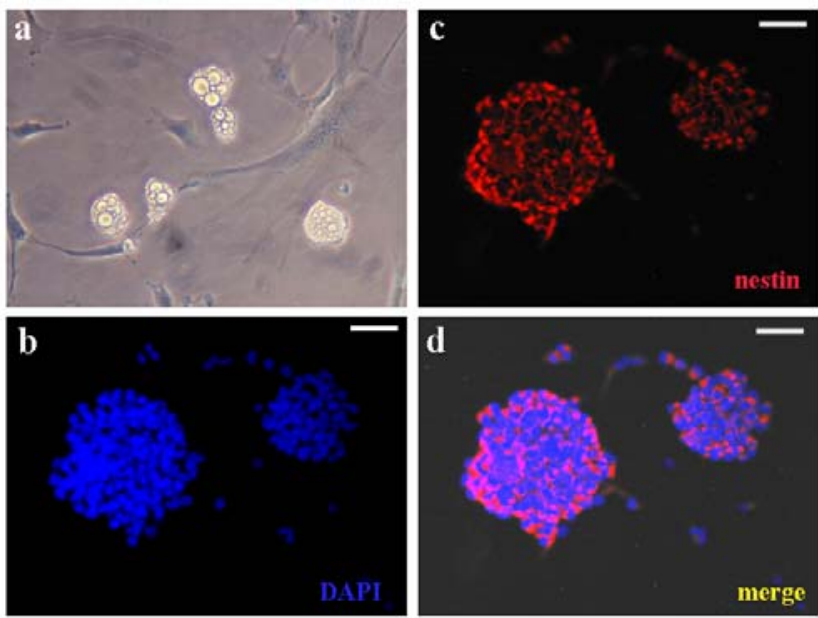

Figure 3. Transition of CNPs from adherent cells to cellular balls in vitro. Adherent CNPs were cultured in growth media and the $45^{\text {th }}$ passage culture was observed under a contrast-phase microscope (200x). It was found that the adherent cells consistently generated floating cells (a, small and bright cellular balls) as observed in primary cell cultures. These newly generated cells grew into cellular balls that were stained with DAPI (b). The cellular ball expressed nestin in the cytosol (c) as merged into DAPI image (d). Bar $=50 \mu \mathrm{m}$. 

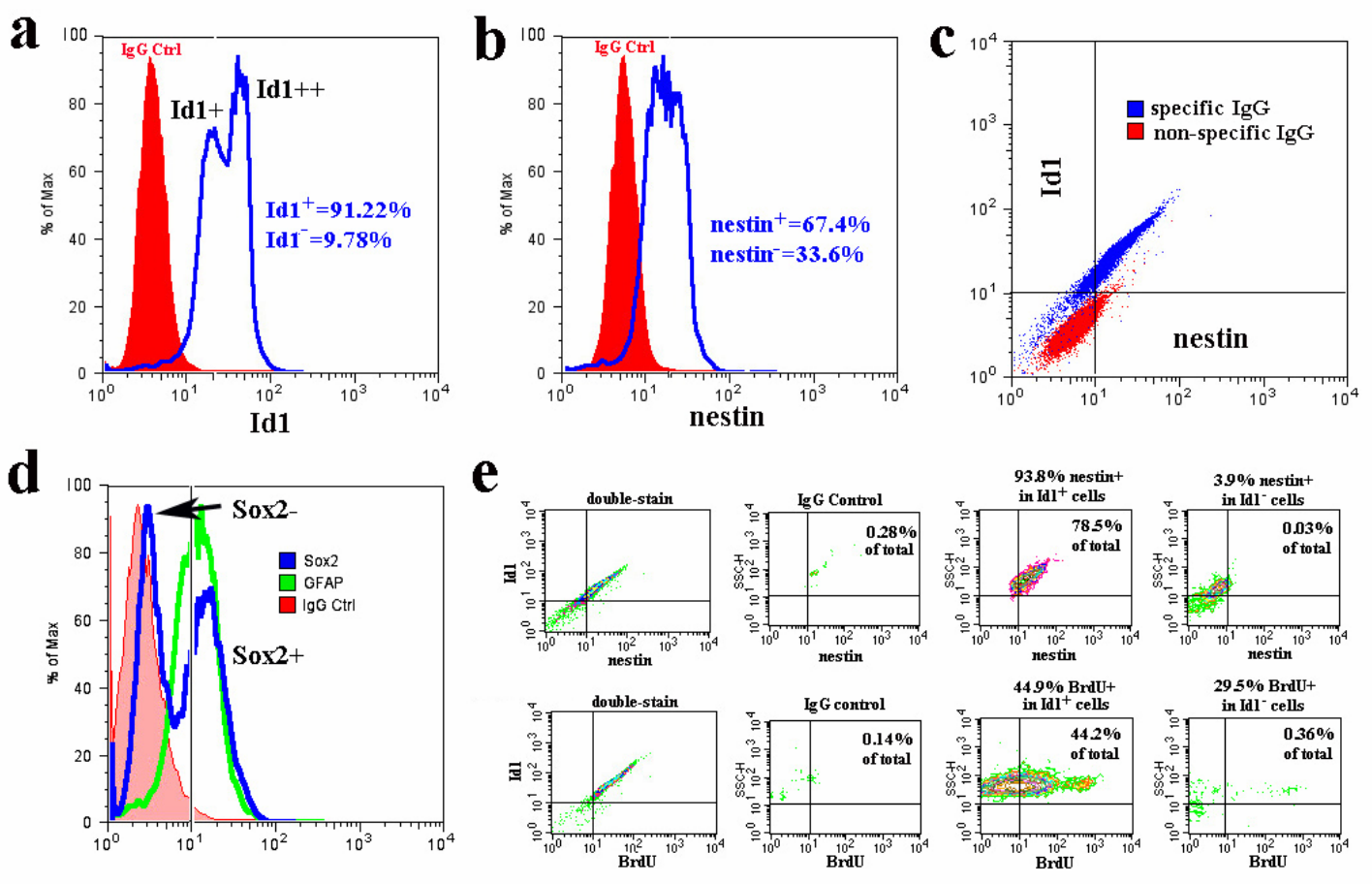

Figure 4. Analysis of CNP marker expression in clone \#5. FACS analysis on Id1, nestin, Sox2, and GFAP in CNPs at 4 day cultures using single- or double-staining procedures as described in the Materials and Methods. Clone \#5 CNPs expressed both Id1 (a) and nestin (b) in the same subpopulation (c). Similarly, clone \#5 CNPs expressed both Sox2 and GFAP (d). It is noted that there were Sox $2^{+}$and Sox2 cells (d) in clone \#5 which is derived from a single CNP and the same applies to Id 1 [ld1 $1^{-}$Id $1^{+}$, and Id $1^{++}$cells in (a)]. Further analyses on Id1 and nestin expressing cells using CellQuest-Pro software indicate that in these Id $1^{+}$cells $\left(91.22 \%\right.$, a), approximately $93.8 \%$ were nestin ${ }^{+}$which account for $78.5 \%$ of total cells and in Id1 cells $\left(9.78 \%\right.$, a), approximately $3.9 \%$ were nestin ${ }^{+}$which account for $0.03 \%$ of total cells compared with IgG control which shows $0.28 \%$ false nestin ${ }^{+}$cells (due to background stain of antibody). Similarly, in these Id $1^{+}$cells $(91.22 \%$, a), approximately $44.9 \%$ were $\mathrm{BrdU}^{+}$which account for $44.2 \%$ of total cells and in Id1 ${ }^{-}$cells $(9.78 \%)$, approximately $29.5 \%$ were BrdU $^{+}$which account for $0.36 \%$ of total cells compared with IgG control which shows $0.14 \%$ false $\mathrm{BrdU}^{+}$cells (due to background stain of antibody). IgG Ctrl, non-specific lgG control. These data, taken together, suggest that Id1 and nestin-double positive cells are more active in proliferation than Id1 and nestin-double negative cells.

\section{Induction of hair cell and supporting cell markers from clonal progenitors}

It is questioned whether CNPs from clonal cultures are capable of differentiating into distinct phenotypes. To answer this question, we used growth factors involved in cochlear development, namely SERB (DMEM/F12+1\%N2+SERB) in combination or individually, for induction of hair cell marker expression. Clonally isolated progenitors grown for 5 passages in growth media were incubated with SERB for 4 days and then harvested for evaluation of hair cell marker expression by FACS. Concentrations of SHH, EGF, RA, and BDNF used in this study are based upon the referenced studies $(5,10,18,19)$. It was found that hair cell markers were significantly induced in CNPs with SERB incubation whereas they were less so in single- or double-factor incubation for 4 days (Fig. $5 \mathrm{a}-\mathrm{f})$. To study whether CNPs from clonal cultures are capable of differentiating into supporting cells, cells were treated as above and evaluated for expression of $\mathrm{p} 27^{\mathrm{kip} 1}$ and $\mathrm{S} 100 \mathrm{~A}$ which are expressed in supporting cells (20) but not hair cells. It was found that SHH induced the expression of these two supporting cell markers (Fig. 5g-h) but not SERB, EGF, BDNF, and bFGF. RT-PCR demonstrated that SHH upregualted the expression of supporting cell markers (Fig. 5i, p27kip1, cytokeratin 18, and collagen IV alpha 6). To confirm this, RT-PCR was performed for evaluation of each factor's effect on hair cell markers. The similar experiments were performed as above and cells were harvested for RTPCR. As expected, SERB incubation resulted in an 

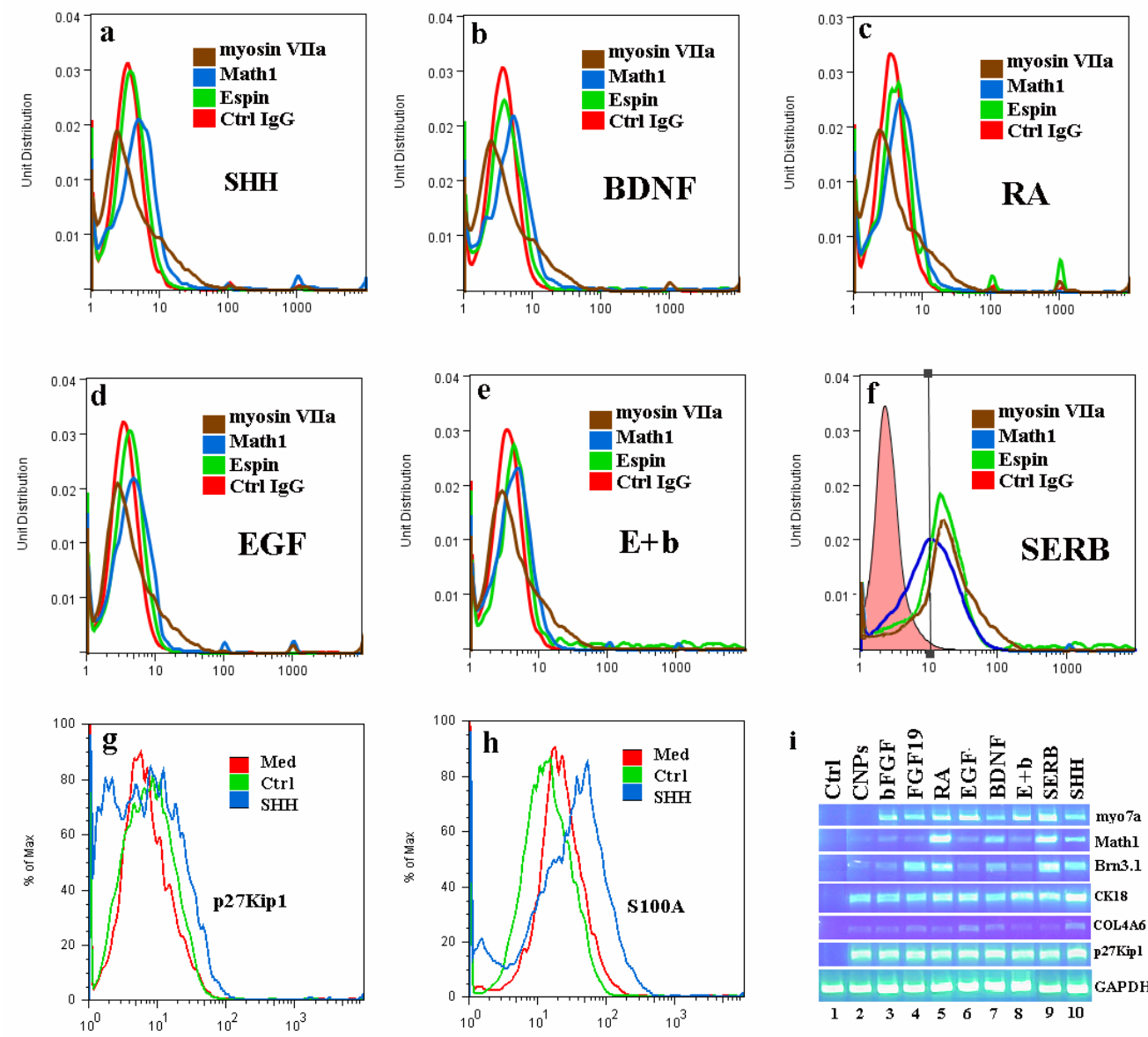

Figure 5. Induction of hair cell markers in CNPs by individual factors or combined factors. CNPs were incubated with individual factors alone or in combination for 4 days and harvested for examination of hair cell markers by FACS. Individual factors alone (a-d) or EGF+bFGF (e) had limited effects on the induction of hair cell markers in CNPs. However, factors in combination (SERB) had significant influence on the expression of hair cell markers (f). SHH promoted the expression of p27 $7^{\text {kip1 }}$ and S100A (supporting cell markers, g-h). By RT-PCR, SHH increased the expression of supporting cell markers p27 $7^{\text {Kip1 }}$, ctyokeratin 18 (CK18) and collagen IV alpha 6 (COL4A6) in a 4-day cell cultures (i). Similarly, SERB combination appeared to play a role in the induction of multiple hair cell markers (i, lane 9) compared with individual factors (i, lanes 2-7) or dual factors (i, lane 8). PCR control ( $i$, lane 1) was negative when mRNA samples were omitted. GAPDH, abbreviation of Glyceraldehyde 3-phosphate dehydrogenase; Med, medium; Ctrl, control.

increased expression of mRNAs for multiple hair cell markers (myosin VIIa, Math1, and Brn3.1). The representative data are presented in Fig. 5i.

\section{Differentiation of clonal progenitors into hair cell- or neuron-like cells}

As expected, differentiation occurred in step 2, with the appearance of neuron-like and cobblestonelike (Fig. 6A). By immunohistochemistry, cobblestone-like cells expressed myosin VIIa and Math1 (Fig. 6C). Remarkably, some cells showed certain mature morphology with espin and F-actin in hair bundle-like morphology (Fig. 6E). Semiquantitative RT-PCR revealed upregulation of hair cell markers (myosin VIIa, Math1, Brn3.1, and epsin) and sensory neuron cell markers (neurogenin 1,
NeuroD, and Brn3a) at the end of step 2 relative to cultures 4 days into step 1, while nestin expression decreased in step 1 and remained low at the end of step 2 relative to progenitor cultures in growth media (Fig. 6B). Continued expression of nestin by RT-PCR after step 2 may reflect persistence of some undifferentiated progenitors in cultures. Differentiated cells were nestin negative by immunohistochemistry (data not shown). Some cells at the end of step 2 clearly expressed both myosin VIIa and Math1 while others expressed both $63^{-}$ tubulin and MAP2 by immunohistochemistry (Fig. $6 \mathrm{C})$. We used FACS on 10,000 cells to quantify the fraction of neural and hair cells: $35.8 \pm 4.6 \%(\mathrm{n}=3)$ were both myosin VIIa and Math1 positive and $12.4 \pm 2.3 \%$ of cells $(n=3)$ were both 83 -tubulin and 


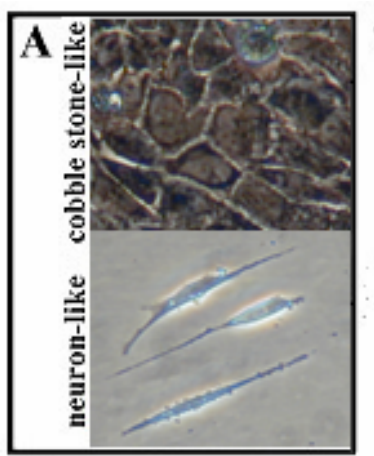

B
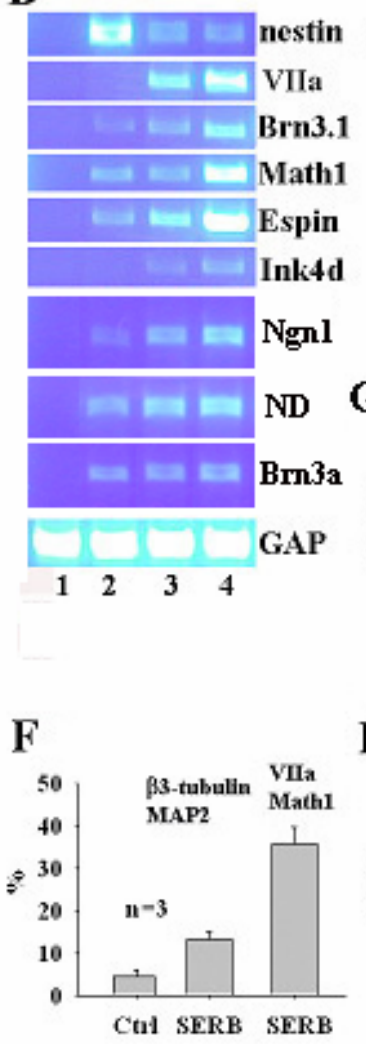

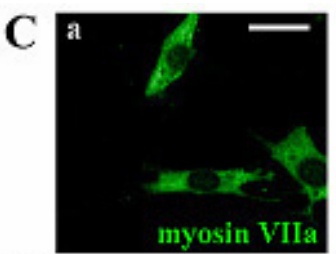

D d

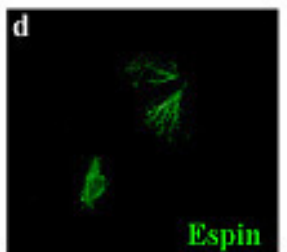

E $g$
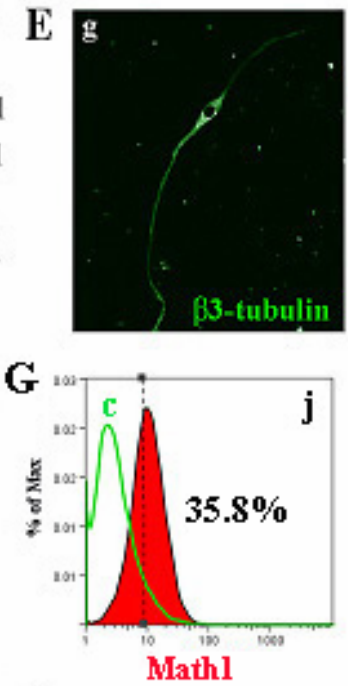

H

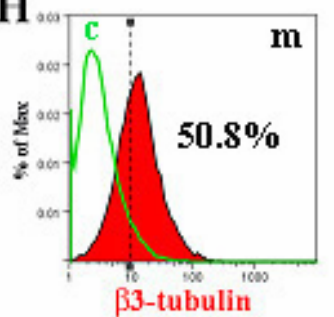

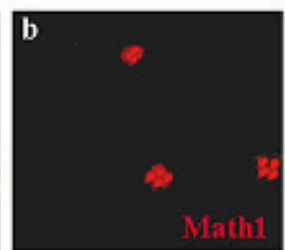
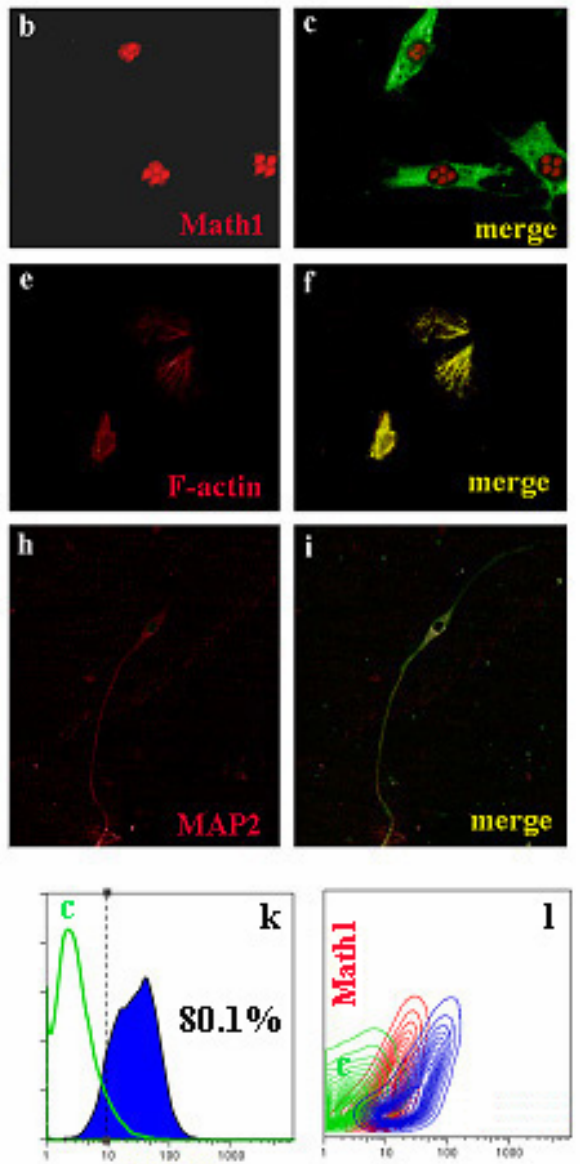

myosin VIIa

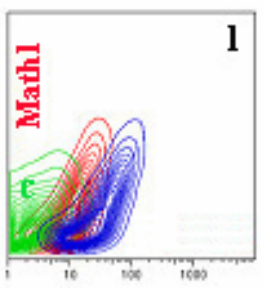

myosin VIIa
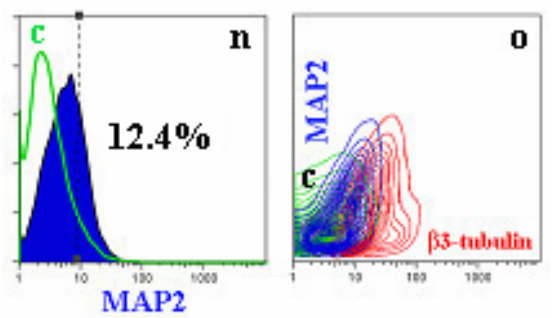

Figure 6. Primed CNPs differentiate into hair cell- and neuron-like cells upon withdrawal of SERB. Two distinct phenotypes (cobblestone-like and neuron-like) were found in cell cultures 7 days following withdrawal of SERB (A). The expression of myosin VIla (VIla), Brn3.1, Math1, espin, p19 ${ }^{\text {Ink4d }}$ (Ink4d), neurogenin 1 (Ngn1), NeuroD (ND), and Brn3a mRNA transcripts was upregulated but nestin downregulated at the end of step 2 (lane 4) compared with those in original CNPs (lane 2) and 4-day cultures with treatment of SERB (lane 3) by RT-PCR (B, Lane 1 as a negative control by omitting RNA sample). The expression of myosin VIla (a, green) and Math1 (b, red) was coexpressed (c) in some columnar or spindle cells $(C)$. The expression of espin (d, green) and F-actin (e, red) was occasionally shown (f, yellow) in hair bundle-like structures (D). 33 -tubulin ( $g$, green) and MAP2 ( $h$, red) were co-expressed (i, yellow) in neuron-like cells (E). Approximately $35.8 \pm 4.6 \%$ of cells were myosin VIla and Math1 double positive (G, $\mathrm{j}-\mathrm{I})$ in cultures at the end of step 2 whereas approximately $12.4 \pm 2.3 \%$ of cells were $\beta 3$-tubulin and MAP2 double positive $(\mathrm{H}, \mathrm{m}-\mathrm{o})$ at the end of step 2. c represents non-specific IgG control or non-differentiated CNPs (j-o). Ctrl, control (no SERB induction); bar=10 $\mu \mathrm{m}$ applying to a-i.

MAP2 positive (Fig. 6F). The difference of \% positive cells between immunohistochemistry and FACS is explained by the fact that the two blind observers counted only highly positive cells, while FACS includes all positive cells regardless of expression levels. Hair cell differentiation was accompanied by upregulation of p19Ink4d expression by semiquantitative RT-PCR (Fig 6B). p19 Ink4d is a transcription factor that prevents cells from entering the cell cycle again (21). 


\section{Discussion}

It is clear in this study and others $(1,2)$ that CNPs exist in the postnatal organ of Corti in mammalians. However, they disappear by 3 weeks after birth (3). It is not clear why this occurs in the organ of Corti of the cochlear system but not in the utricle of the vestibular system in which both stem cells or progenitors share similar features $(3,5)$. This may be attributed to the proliferation inhibition of CNPs in the organ of Corti but not in the vestibular system because knockout of $R b 1$ in mice led to the production of extra hair cells in the organ of Corti (8) but not in the vestibular system. These studies prompt us to examine whether CNPs are self-renewable when the Rb protein function is disabled by SV40 large T antigen.

Indeed, CNPs from the P1 Immortomouse organ of Corti exhibit the property of self-renewal in an unlimited manner in vitro under the culture conditions using growth media that are consisted of DMEM/F12, 1\%N2, $20 \mathrm{ng} / \mathrm{mL}$ of EGF, and $20 \mathrm{ng} / \mathrm{mL}$ of bFGF. Self-renewal capability of cochlear stem cells or neural progenitor from non-Immortomouse organ of Corti has recently been shown by Oshima et al., (3) and ours (4) but how long it lasts remains to be elucidated. This study indicates that SV40 T large antigen plays a role in self-renewal of cochlear neural progenitors.

In SV40 $\mathrm{T}$ large antigen positive cells, BrdU incorporation studies indicate that cells are highly active in proliferation. These highly proliferating cells are positive for Id 1 and nestin proteins. Id 1 has been shown in our previous study for cellular proliferation of rat sensory epithelial cells (15). In primitive cochlear neural progenitors, nestin is a marker for immature neural epithelial cells, which is co-expressed with Id1 (Id1 positive cells are 94\% nestin positive and $45 \%$ of them BrdU positive) (4) whereas in multiple passaged $\mathrm{SV}^{+} 0^{+}$clonal progenitors, nestin and Id1 remain almost at the same levels as those primitive progenitors and cells are highly proliferative (44.9\% of cells BrdU positive with Id1 positive cells). It is interesting to know that this clonal lineage contains two distinct subpopulations, Sox $2^{+} / S_{0 \times} 2^{-}$cells, which may represent distinct pro-neuron or pro-sensory subpopulations. Consistent with Sox 2 expression data, GFAP is also positive in some of those cells. Since $S o x 2$ and GFAP are highly expressed in NPCs, we would assume that $S_{0 x 2^{+}}$and $\mathrm{GFAP}^{+}$cells are pro-neuron progenitors in this culture system. The pro-neuron progenitors may differentiate into sensory neurons after SERB induction because cochlear sensory neuron markers are upregulated at the end of step 2.

One question we have here is that whether highly proliferative cells, driven by SV40, are able to differentiate at $37^{\circ} \mathrm{C}$ (a body temperature) under cultured conditions. If such, CNPs with tsSV40 may be used for cell replacement in degenerative hearing disorders. As expected, CNPs at $37^{\circ} \mathrm{C}$ responded to induction factors (SERB) as those progenitors or cochlear stem cells from the wild-type mice (4). Furthermore, CNPs from the Immortomouse P1 organ of Corti can be used for further differentiation toward hair cells with some mature morphological features such as hair cell bundles. Our data indicate that CNPs from clonal cultures are multipotent, capable of differentiating into neural and hair cell phenotypes. However, there is a concern for using CNPs as cell replacement in hearing disorders. As CNPs contain an oncogene SV40, one would think that these cells will be prone to tumorigenesis when the proliferative potential of CNPs is combined with tumor-inducing factors or environments. This issue has to be resolved before CNPs can be used for cell therapy in hearing disorders.

Unlike CNPs from wild-type mice, CNPs from the Immortomouse are capable of self-renewal in relatively simple media such as $\mathrm{DMEM} / \mathrm{F} 12+1 \% \mathrm{~N} 2+\mathrm{EGF}+\mathrm{bFGF}$. This is the first demonstration that tsSV40 drives self-renewal of CNPs in vitro and tsSV40 transduced progenitors are capable of differentiating into hair cell- and neuron-like cells under the guidance of a group of ear development-specific growth factors: SERB. Cocktail treatment is recently known for its transduction power. For example, transduction of mouse embryo fibroblasts with 4 genes or oncogenes (Oct4, Sox2, c-myc, and klf4) generated cells closely resembling embryonic stem cells (22-24).

On the one hand, tsSV $40^{+}$progenitors express multiple neuroepithelial cell markers: nestin, Sox2, GFAP, and Musashi, a marker profile similar to that of NPC neurospheres. On the other hand, tsSV40 progenitors express espin and Pax2. In addition, Kalinec and coworkers demonstrated that tsSV40 ${ }^{+}$ organ of Corti cell lines from the Immortomouse P14 organ of Corti express organ of Corti protein 2 (OCP2) and epithelial cell tight junction protein $\mathrm{ZO}^{-}$ 1 (2). It is noted that these progenitors are multipotent which have the potential to differentiate into neuronal cells or sensorineural epithelial cells and supporting cells under the guidance of SERB. Supporting cells are thought as progenitor hair cells 
which trans-differentiate into hair cells under circumstances (25).

A reason for using SERB is that CNPs grown in growth media for 14 days followed by withdrawal of growth factors (EGF+bFGF) for 7 days did not appear to induce sufficient differentiation as one would expect. Malgrange at al showed that withdrawal of mitogenic growth factors from growth media initiated limited differentiation of progenitor cells in rats (1). In organotypic cultures, none of the individual growth factors alone are able to induce hair cell regeneration. This may be due to limited effects of individual factors on removal of negative forces for cellular proliferation: Hes1, Rb1, and p19ink4d $(8,21,26)$. The combination of multiple factors such as SERB appears to be potent for removal of cellular proliferation inhibition in vitro. Oncogene SV40 alone promotes the self-renewal of cochlear neural progenitors. $\mathrm{SV}_{4} 0^{+}$progenitors cultured at $37^{\circ} \mathrm{C}$ may partially inactivate but not fully inactivate the activity of SV40, which appears to be sufficient for driving progenitor renewal in the presence of EGF and bFGF but not interfering with cellular differentiation in the absence of EGF and bFGF.

One of the key actions of SERB is its induction of the Math1 mRNA or protein expression in cochlear neural progenitors. Math1 is known to play an important role in the initial cochlear sensory epithelial cell generation (27). Introduction of Math1 cDNA into deafened organ of Corti has been shown to generate new hair cells in the guinea pigs (28-30), which suggests that upregulation of the Math1 gene by relevant growth factors such as SERB may lead to the generation of new hair cells in deafened cochleas.

In summary, the identification of CNPs from the Immortomouse postnatal organ of Corti is the first step to study cell replacement in degenerative hearing disorders. Using these progenitors, we have shown that they are capable of differentiating into hair cell-, neuron-, and supporting cell-like phenotypes under the influence of SERB. This study implies that renewable progenitors can be induced by transduction of SV40 oncogene and potentially useful for cell replacement in degenerative hearing disorders.

\section{Acknowledgments}

This study is in part supported by the NIDCD R01 DC008165, P30DC04660, NCI R03CA107989, and the Academic Health Center seed grant. We would like to express our thanks to Dr. Jin Xiao at the Neurosurgery Department, University of Minnesota for her help in NPC culture as well as Mr. Danial Guire for his editorial assistance in the preparation of this manuscript.

\section{References}

1. Malgrange B, Belachew S, Thiry M, et al. Proliferative generation of mammalian auditory hair cells in culture. Mech Dev 2002;112:79-88.

2. Kalinec F, Kalinec G, Boukhvalova M, Kachar B. Establishment and characterization of conditionally immortalized organ of Corti. Cell Biol Int 1999;23:175-84.

3. Oshima K, Grimm CM, Corrales CE, et al. Differential distribution of stem cells in the auditory and vestibular organs of the inner ear. J Assoc Res Otolaryngol 2007;8:18-31.

4. Lin J, Feng L, Fukudome S, et al. Cochlear stem cells/progenitors and degenerative hearing disorders. Curr Med Chem 2007;14:2937-43.

5. Li H, Liu H, Heller S. Pluripotent stem cells from the adult mouse inner ear. Nat Med 2003;9:1293-9.

6. Chen P, Johnson JE, Zoghbi HY, Segil N. The role of Math1 in inner ear development: Uncoupling the establishment of the sensory primordium from hair cell fate determination. Development 2002;129:2495-505.

7. Kim HY, Ahn BY, Cho Y. Structural basis for the inactivation of retinoblastoma tumor suppressor by SV40 large T antigen. EMBO J 2001;20:295-304.

8. Sage C, Huang M, Karimi K, et al. Proliferation of functional hair cells in vivo in the absence of the retinoblastoma protein. Science 2005;307:1114-8.

9. Liu W, Li G, Chien JS, et al. Sonic hedgehog regulates otic capsule chondrogenesis and inner ear development in the mouse embryo. Dev Biol 2002;248:240-50.

10. Lefebvre PP, Malgrange B, Staecker H, et al. Retinoic acid stimulates regeneration of mammalian auditory hair cells. Science 1993;260:692-5.

11. Feng L, Fukudome S, Wang D, et al. BDNF increases the survival of rat sensory epithelial cells via the PI3K/AKT and NF-kB/BCL-2 signaling pathways submitted 2008.

12. Sam TN, Xiao J, Roehrich H, et al. Engrafted neural progenitor cells express a tissue-restricted reporter gene associated with differentiated retinal photoreceptor cells. Cell Transplant 2006;15:147-60.

13. Jat PS, Noble MD, Ataliotis P, et al. Direct derivation of conditionally immortal cell lines from an $\mathrm{H}-2 \mathrm{~Kb}-\mathrm{tsA} 58$ transgenic mouse. Proc Natl Acad Sci U S A 1991;88:5096100.

14. Ozeki M, Duan L, Obritch W, Lin J. Establishment and characterization of progenitor hair cell lines in rats. Hear Res 2003;179:43-52.

15. Ozeki M, Hamajima Y, Feng L, et al. Id1 induces the proliferation of cochlear sensorineural epithelial cells via the NF-kB/cyclin D1 pathway in vitro. J Neurosci Res 2007;85:515-24.

16. Molofsky AV, Pardal R, Iwashita T, et al. Bmi-1 dependence distinguishes neural stem cell self-renewal from progenitor proliferation. Nature 2003;425:962-7.

17. Li H, Corrales CE, Edge A, Heller S. Stem cells as therapy for hearing loss. Trends Mol Med 2004;10:309-15. 
18. Kondo T, Johnson SA, Yoder MC, et al. Sonic hedgehog and retinoic acid synergistically promote sensory fate specification from bone marrow-derived pluripotent stem cells. Proc Natl Acad Sci U S A 2005;102:4789-94.

19. Alderson RF, Alterman AL, Barde YA, Lindsay RM. Brain-derived neurotrophic factor increases survival and differentiated functions of rat septal cholinergic neurons in culture. Neuron 1990;5:297-306.

20. Doetzlhofer A, White PM, Johnson JE, et al. In vitro growth and differentiation of mammalian sensory hair cell progenitors: a requirement for EGF and periotic mesenchyme. Dev Biol 2004;272:432-47.

21. Chen P, Zindy F, Abdala C, et al. Progressive hearing loss in mice lacking the cyclin-dependent kinase inhibitor Ink4d. Nat Cell Biol 2003;5:422-6.

22. Okita K, Ichisaka T, Yamanaka S. Generation of germline-competent induced pluripotent stem cells. Nature 2007;448:313-7.

23. Takahashi K, Okita K, Nakagawa M, Yamanaka S. Induction of pluripotent stem cells from fibroblast cultures. Nat Protoc 2007;2:3081-9.

24. Nakagawa M, Koyanagi M, Tanabe K, et al. Generation of induced pluripotent stem cells without Myc from mouse and human fibroblasts. Nat Biotechnol 2008;26:101-6.

25. Fekete DM, Muthukumar S, Karagogeos D. Hair cells and supporting cells share a common progenitor in the avian inner ear. J Neurosci 1998;18:7811-21.

26. Zheng JL, Shou J, Guillemot F, et al. Hes1 is a negative regulator of inner ear hair cell differentiation. Development 2000;127:4551-60.

27. Bermingham NA, Hassan BA, Price SD, et al. Math1: an essential gene for the generation of inner ear hair cells. Science 1999;284:1837-41.

28. Zheng JL, Gao WQ. Overexpression of Math1 induces robust production of extra hair cells in postnatal rat inner ears. Nat Neurosci 2000;3:580-6.

29. Kawamoto K, Ishimoto S, Minoda R, et al. Math1 gene transfer generates new cochlear hair cells in mature guinea pigs in vivo. J Neurosci 2003;23:4395-400.

30. Izumikawa M, Minoda R, Kawamoto K, et al. Auditory hair cell replacement and hearing improvement by Atoh1 gene therapy in deaf mammals. Nat Med 2005;11:271-6.

\section{Supplemental Data}
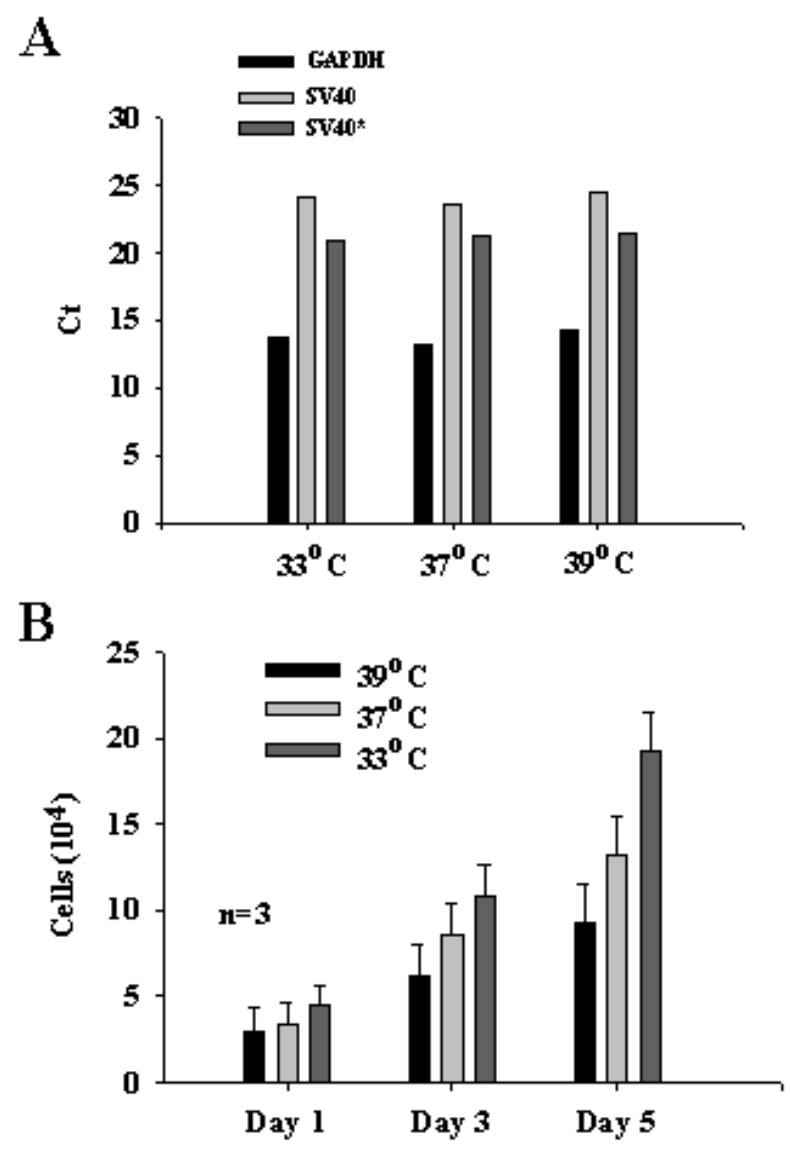

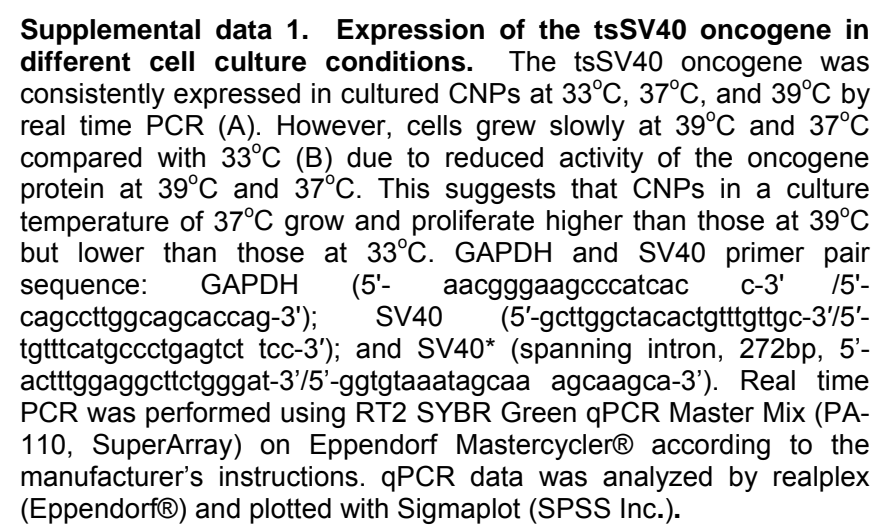

